\title{
Peripheral Odour Perception by Adult Aphid Forms with the Same Genotype but Different Host-Plant Preferences
}

\author{
JIM HARDIE, $* \ddagger$ J. H. VISSER, $\dagger$ P. G. M. PIRON $\dagger$ \\ Received 16 May 1994
}

www. olfacts.nl

\begin{abstract}
As adults, the summer (alate virginopara) and autumn (gynopara) winged forms of the black bean aphid, Aphis fabae, utilise different host plants, the bean Vicia faba, and the spindle tree, Euonymus europaeus, respectively. Electroantennograms (EAGs) were recorded from the antennae of these aphid forms, reared from a single clone, to thirty five plant volatiles and to paraffin oil extracts from leaves of the two host plants. The EAG peak responses were normalised against the adjacent responses to a standard stimulus $((E)-2$-hexenal at $\log 2$ dilution). The comparison of different parameters of the EAG responses, i.e. peak, rise and decay, reveals that peripheral olfactory perception of most volatiles is not significantly different between these two forms. In addition, the responses of adult wingless virginoparae to thirteen of the plant volatiles show, with one exception, similar peripheral perception. The results indicate that any odour discrimination between the two alternative host plants by their respective colonisers is not perceived at the level of the peripheral olfactory receptors. The EAG response profile of $A$. fabae differs from that of the vetch aphid, Megoura viciae, which also colonises bean.
\end{abstract}

Aphids Aphis fabae Electroantennogram EAG decay EAG rise Euonymus europaeus Gynoparae Host selection Kairomones Odours Olfaction Plant volatiles Semiochemicals Vicia faba Virginoparae

\section{INTRODUCTION}

Although earlier studies concluded that aphids did not use odour cucs for host-plant location (Kennedy et al., 1959), it has recently become clear that they do, although visual cues are still considered important ( $\AA$ hman et al., 1985). Aphid antennae possess olfactory receptors which respond to plant volatiles (Bromley and Anderson, 1982; Yan and Visser, 1982; Wohlers and Tjallingii, 1983; Dawson et al., 1987; Van Giessen et al., 1992; Wadhams, 1990). Aphids respond behaviourally in laboratory situations to plant odours both walking (Pettersson, 1971; Visser and Taanman, 1987; Nottingham et al., 1991) or flying (Nottingham and Hardie, 1993) and in the field (Chapman et al., 1981). Single cell recording techniques offer the opportunity to observe the responses of individual sensory neurones to odour stimuli (e.g. Bromley and Anderson, 1982) while electroantennogram (EAG) recordings provide an overview of total antennal respon-

\footnotetext{
*Department of Biology, Imperial College at Silwood Park, Ascot, Berks SL5 7PY, U.K.

†Research Institute for Plant Protection (IPO-DLO), P.O. Box 9060, $6700 \mathrm{GW}$ Wageningen, The Netherlands.

†To whom all correspondence should be addressed.
}

siveness (Boeckh et al., 1965). In addition, detailed analysis of the time course of the EAG allows conclusions to be drawn concerning the transport of volatiles to the receptor sites in the dendritic membranes and the process of inactivation following stimulation (Dickens et al., 1993). In aphids there are two main types of olfactory organs on the antennae, the distal (on segment six) and the proximal (on segment five) primary rhinaria which are present in larval and adult forms, and secondary rhinaria, primarily located on segment three, which appear at the final moult and occur in alatae but are less numerous or absent in apterae (Pickett et al., 1992).

Aphid life cycles can be extremely complex, involving parthenogenetic and sexual reproduction as well as phenotypically different parthenogenetic females (Hille Ris Lambers, 1966; Moran, 1992). In host-alternating (heteroecious) species, such as the black bean aphid, Aphis fabae Scop., the seasonal cycle moves between a primary (winter), often woody host and a secondary (summer), herbaceous host. Sexual reproduction is associated with the primary host and parthenogenetic reproduction with the secondary host. Summer parthenogenetic females are determined as winged adults (alate virginoparae), by nutritional factors and high 
population densities, or wingless adults (apterous virginoparae) under more isolated conditions. The short days of autumn induce the appearance of a generation of winged females (gynoparae) which fly to the winter host where they give birth to the sexual females (oviparae). Short days also induce males which mate with the oviparae on the primary host. For A. fabae the summer females, both alatae and apterae, prefer to feed and reproduce on the summer host (e.g. broad bean, Vicia faba L.) while the gynoparae prefer the winter host (the spindle tree, Euonymus europaeus L.) (Kennedy and Booth, 1951; Hardie, 1980, 1981; Hardie and Glascodine, 1990). This preference associated with host alternation can also be seen in other aphid species e.g. Rhopalosiphum padi (Dixon, 1981) and Cryptomyzus species (Guldemond, 1990). Thus, the polyphenism exhibited in these clonal insects encompasses not only morphology but also host-plant preference. The present experiments set out to discover whether there are differences between the female morphs of $A$. fabae in the peripheral detection of plant volatiles which might be associated with the different host-plant preference.

\section{METHODS AND MATERIALS}

Insects

A clone of Aphis fabae originally established by Kennedy and Booth (1951) was cultured on tick bean ( $V . f a b a)$ seedlings at $15^{\circ} \mathrm{C}$ in Gallenkamp incubators. Apterous and alate virginoparae were reared under long-day conditions (LD 16:8), apterous adults developed in sparse populations while alatae were induced by crowding young larvae. Transfer of long-day insects to short days induced the development of the alate gynoparae (see Hardie, 1980). Alate virginoparae and gynoparae were used shortly after first take-off $(<24 \mathrm{~h}$ old) while pre-reproductive apterae were selected $(<72 \mathrm{~h}$ old).

\section{Electroantennogram recordings}

Antennal preparation involved amputation of the left antenna at the base and severing the anterior part of the head capsule at eye level. A glass capillary, filled with $0.1 \mathrm{M} \mathrm{KCl}$, formed the indifferent electrode and was inserted into the head so that the tip lodged close to the base of the intact antenna. The extreme tip of the sixth antennal segment was then removed and the cut end sleeved with a $0.1 \mathrm{M}$ KCl-filled recording electrode. Chlorided-silver wires were inserted into the electrodes and connected to a Grass P16D amplifier via an HIP16A input probe. Amplified signals were observed on a Philips PM3302 storage oscilloscope and recorded using a Krenz TRC 4010 transient recorder attached to an Estate AT386 computer (for further details see Visser and Piron, 1994a, b, c).

The electrical recordings started $4 \mathrm{~s}$ prior to the $2 \mathrm{~s}$ odour stimulus injection and continued for $14.5 \mathrm{~s}$ afterwards. At the air flow rate used, the odour stimulus reached the antennal preparation $0.5 \mathrm{~s}$ after injection but the time of injection was taken as second 0.0 . The data were analysed by software developed in Asyst 3.1 (Visser and Piron, 1994a, b, c). The originat signals were smoothed, corrected for DC drift (in relation to prestimulus signal) and the parameters examined were:

1. Peak response, the largest negative potential recorded in seconds $0.0-2.5$ from the onset of stimulus injection in $\mu \mathrm{V}$.

2. Mean Response, the mean potential recorded in seconds $1.5-2.0$ in $\mu \mathrm{V}$.

3. Rise, the mean potential reached in seconds $0.0-1.0$ relative to the Mean Response in \%.

4. Decay, the mean dccrcasc in potential rcached in seconds 2.5-3.5 relative to the Mean Response in \%.

The Peak responses were normalised and expressed as a percentage in relation to adjacent EAG peaks produced in response to the standard odour $((E)$-2-hexenal, see below).

\section{Odour stimuli, presentation and protocol}

The chemicals were obtained from commercial suppliers or specially prepared (Table 1) and comprised natural plant volatiles. All chemicals were $\geqslant 95 \%$ pure except for heptanonitrile $(92 \%), \quad(E, E)-\alpha$-farnesene $(92 \%)$ and $(E)-\beta$-farnesene $(58 \%)$ which also contained $35 \% \alpha$-farnesene isomers. These chemicals were dissolved in paraffin oil (Merck, Uvasol), $10 \mu 1$ in $1 \mathrm{ml}$ ( $\log 2$ dilution). Where other concentrations were used, they are stated.

Paraffin oil solution $(25 \mu \mathrm{l})$ was pipetted onto a $0.8 \times 6 \mathrm{~cm}$ piece of filter paper (Schleicher \& Schuell $589^{2}$, Germany) and the paper inserted into a glass Pasteur pipette. The pipette was attached to an air line and the volatile delivered, over a $2 \mathrm{~s}$ interval, into the main air flow over the antennal preparation. The air was purified by passage through Chrompack moisture and charcoal filters and then re-humidified by bubbling through de-ionised water. Air flow was controlled at $1800 \mathrm{ml} / \mathrm{min}$ for the main flow which was directed over the preparation by a $1 \mathrm{~cm}$ dia. glass tube (i.e. $40 \mathrm{~cm} / \mathrm{s}$ ). The tip of the Pasteur pipette containing the volatile was inserted through a small hole in the wall of the glass tubing and air forced through at $60 \mathrm{ml} / \mathrm{min}$. Air flow was controlled by Brooks mass flow controllers and the stimulus via a 3-way solenoid valve.

The antennal preparations proved delicate, EAG responses tended to decrease with time and the preparations were short-lived $(<20 \mathrm{~min})$. There were also differences in EAG amplitude between antennae. To overcome this variability all test stimuli were bracketed by a standard stimulus with $(E)$-2-hexenal (at a dilution of $10 \mu \mathrm{l}$ in $1 \mathrm{ml}$ paraffin oil). EAG peak responses could then be expressed in terms relative to the adjacent standards. There were $c .30 \mathrm{~s}$ between stimulus presentations. 
Extraction of bean and spindle volatiles

Thirty g, wet weight, of broad bean ( $V . f a b a, c v$. Sutton Dwarf) and spindle (E. europaeus) leaves were homogenised in $100 \mathrm{ml}$ of de-ionised water using a Waring blender. The mixture was filtered through glass wool, $50 \mathrm{ml}$ of the filtered aqueous extract was added to $50 \mathrm{ml}$ paraffin oil, shaken and then centrifuged at $7000 \mathrm{rpm}$ for $10 \mathrm{~min}$. The paraffin oil/volatile layer was, thus, separated from water and $25 \mu 1$ used to stimulate EAGs without dilution.

\section{RESULTS}

Examples of EAGs recorded from an alate virginoparous $A$. fabae in response to the $(E)$-2-hexenal standard and $(Z)-3$-hexenol-1 are shown in Fig. 1. The peak responses to the standard, in absolute terms, of the different aphid forms examined were similar:

TABLE 1. Key to volatiles

\begin{tabular}{|c|c|c|}
\hline Chemical & $\begin{array}{c}\text { Code } \\
\text { number }\end{array}$ & Source \\
\hline \multicolumn{3}{|l|}{ Green leaf volatiles } \\
\hline (E)-2-Hexenal & 2 & Roth \\
\hline (E)-2-Hexenol-1 & 4 & Roth \\
\hline (Z)-3-Hexenol-1 & 5 & Roth \\
\hline$(Z)-3$-Hexenyl acetate & 11 & Roth \\
\hline Hexanol-1 & 15 & Fluka \\
\hline Hexanal & 24 & Fluka \\
\hline (E)-2-Heptenal & 102 & Aldrich \\
\hline 2-Hexanone & 30 & Fluka \\
\hline 2-Heptanone & 32 & Aldrich \\
\hline \multicolumn{3}{|l|}{ Benzaldehydes } \\
\hline Benzaldehyde & 36 & Roth \\
\hline 2-Methoxybenzaldehyde & 37 & Aldrich \\
\hline 3-Methoxybenzaldehyde & 38 & Aldrich \\
\hline 4-Methoxybenzaldehyde & 39 & Aldrich \\
\hline 2-Hydroxybenzaldehyde & 40 & Roth \\
\hline \multicolumn{3}{|l|}{$\begin{array}{l}\text { Monoterpenes, -alcohols and } \\
\text {-aldehydes }\end{array}$} \\
\hline$(-)-(1 S)-\alpha-$ Pinene & 45 & Fluka \\
\hline$(-)-(1 S)-\beta$-Pinene & 46 & Fluka \\
\hline$(+)-(S)$-Carvone & 49 & Roth \\
\hline$(-)-(R)$-Carvone & 50 & Aldrich \\
\hline$\alpha$-Terpineol & 56 & Roth \\
\hline Linalool & 59 & Fluka \\
\hline Geraniol & 60 & Fluka \\
\hline Nerol & 61 & Aldrich \\
\hline Citronellal & 65 & Roth \\
\hline$(+)$-Citronellol & 66 & Roth \\
\hline \multicolumn{3}{|l|}{ Sesquiterpenes } \\
\hline Sabinene & 69 & Roth \\
\hline$(-)(E)$-Caryophyllene & 70 & Fluka \\
\hline \multicolumn{3}{|l|}{ Farnesenes } \\
\hline$(E, E)-\alpha$-Farnesene & 82 & TNO \\
\hline$(E)-\beta$-Farnesene & 83 & TNO \\
\hline \multicolumn{3}{|l|}{ N-containing compounds } \\
\hline Hexanonitrile & 85 & Aldrich \\
\hline Heptanonitrile & 86 & $\mathrm{ICN} / \mathrm{K} \& \mathrm{~K}$ \\
\hline Butyl isothiocyanate & 89 & Aldrich \\
\hline tert-Butyl isothiocyanate & 90 & Aldrich \\
\hline Allyl isothiocyanate & 91 & Aldrich \\
\hline 3-Butenyl isothiocyanate & 126 & Rothamsted \\
\hline 4-Pentenyl isothiocyanate & 127 & Rothamsted \\
\hline
\end{tabular}
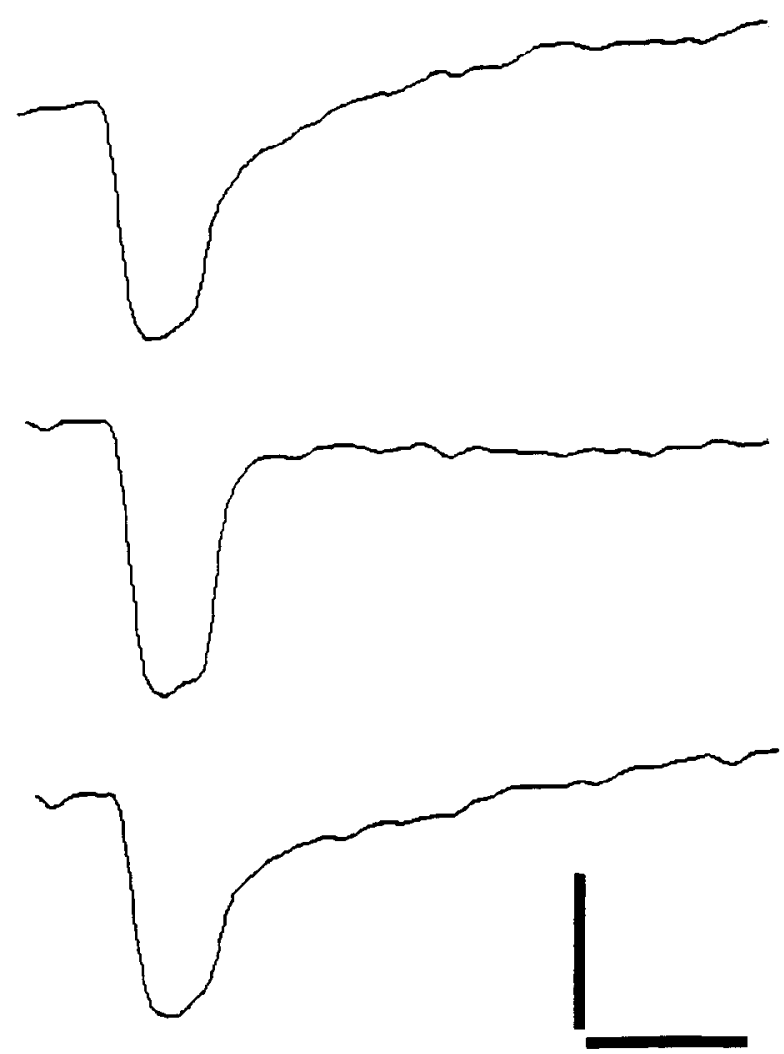

FIGURE 1. Electroantennogram recordings, smoothed and corrected for DC drift, from an alate virginopara to $(Z$ )-3-hexenol-1 (centre) together with responses to the adjacent standard $(E)$-2-hexenal (upper and lower). Chemicals are in $\log 2$ dilution at the source. Scale bars are $100 \mu \mathrm{V}, 4 \mathrm{~s}$.

$270 \pm 90 \mu \mathrm{V}$ for apterae (mean $\pm 95 \% \quad$ C.I.), $220 \pm 60 \mu \mathrm{V}$ for alate virginoparae and $190 \pm 60 \mu \mathrm{V}$ for gynoparae.

Comparison of the normalised EAG peak responses produced by alate virginoparae and gynoparae show that for the standard (compound 2) and thirty four test chemicals the responses are identical (Fig. 2). EAGs elicited to general green leaf volatiles (see Table 1) indicate similar responses to $\mathrm{C} 6$ alcohols and aldehydes (compounds $2,4,5,15,24)$ and to the $C 7$ aldehyde (102) while the acetate (11) and ketones $(30,32)$ evoke smaller responses. The $\mathrm{C} 7$ compounds tested are as effective as or more effective than their $\mathrm{C} 6$ equivalents (102 vs $2 ; 32$ vs 30 ). Of the other compounds tested only the hexanoand heptanonitriles (85 and 86) elicit larger EAGs than the standard. Benzaldehyde, methoxy and hydroxy derivatives $(36,37,38,39,40)$ elicit responses ranging from $40-75 \%$ of the standard while the maximum response from the isothiocyanates is $c .90 \%$ with butyl (89), 3-butenyl (126), 4-pentenyl (127) > allyl (91) > tert butyl (90). The two pinene isomers (45 and 46) and the two carvone isomers ( 49 and 50 ) are not distinguished by the EAG responses while the monoterpene alcohols tend to elicit smaller responses $(56,59,60$ and 61$)$. Citronellal (65) is a more potent stimulus than citronellol (66) and 

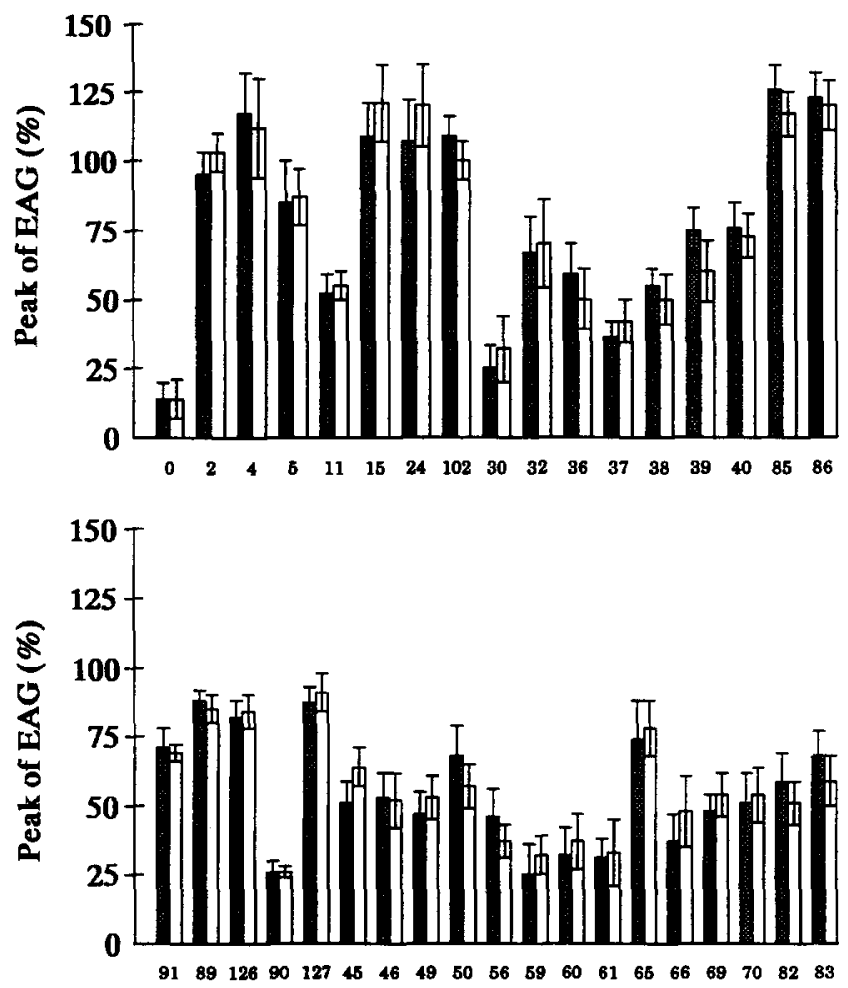

Chemicals at Log Dilution 2

FIGURE 2. Comparison of the normalised EAG peak responses (mean $\pm 95 \%$ C.I.) from alate virginoparae (hatched, $n=11-19$ ) and gynoparae (outline, $n=10-13$ ) to the paraffin oil blank (0) and 35 test volatiles. See Table 1 for key to compounds.

both farnesene isomers ( 82 and 83 ) and the two sesquiterpenes (69 and 70) evoke similar responses.

The transport of the odour molecules to the receptor sites in the dendritic membranes and the subsequent process of their inactivation are reflected in the rise and decay parameters, respectively, of the recorded EAGs. The rise and decay of EAGs from alate virginoparae and gynoparae are similar (Fig. 3). In Fig. 3, compounds 59 and 90 are excluded since the amplitudes of their EAGs

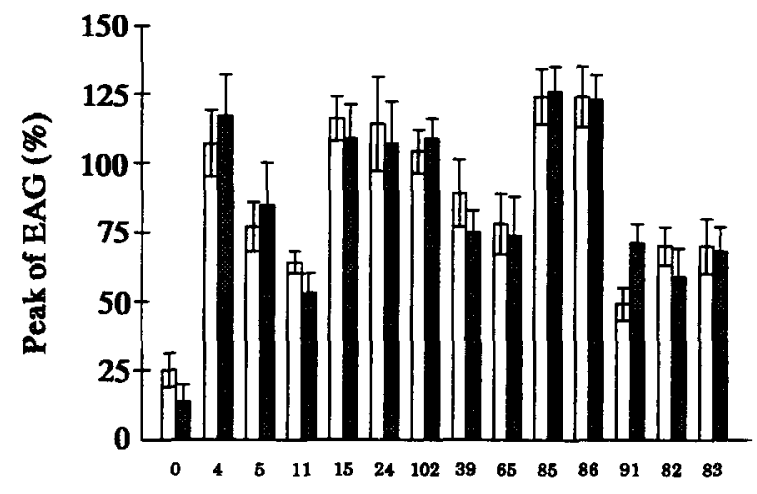

Chemicals at Log Dilution 2

FIGURE 4. Comparison of the normalised EAG peak responses (mean $\pm 95 \%$ C.I.) from apterous (outline, $n=9-15$ ) and alate (hatched, $n=12-19)$ virginoparae to the paraffin oil blank (0) and 13 test volatiles. See Table 1 for key to compounds.

are not different from the paraffin oil blank. For both rise and decay parameters there is a significant correlation with $r=0.88 ; P<0.001$; slope $=0.98 ; n=33$ for rise, and $r=0.90 ; P<0.001$; slope $=1.10 ; n=33$ for decay. Only, $(Z)$-3-hexenol-1 (5), shows rise percentages where the confidence intervals do not overlap while hexanol-1 (15) evokes EAGs with different decay times. Thus, all volatiles tested on alate virginoparae and gynoparae produce EAGs with almost identical shape characteristics.

Thirteen compounds were also tested on antennae from apterous virginoparae and the normalised EAG peak responses are compared with those of alate virginoparae in Fig. 4. Only allyl isothiocyanate (91) shows a significant difference and is lower in the apterae. All recorded rise times overlap with those of the alate virginoparae/gynoparae but two compounds, citronellal (65) and heptanonitrile (86), produce significantly different decay parameters.

The comparison between rise and decay parameters is complicated by the fact that these may differ in EAGs produced by different dilutions of the same volatile
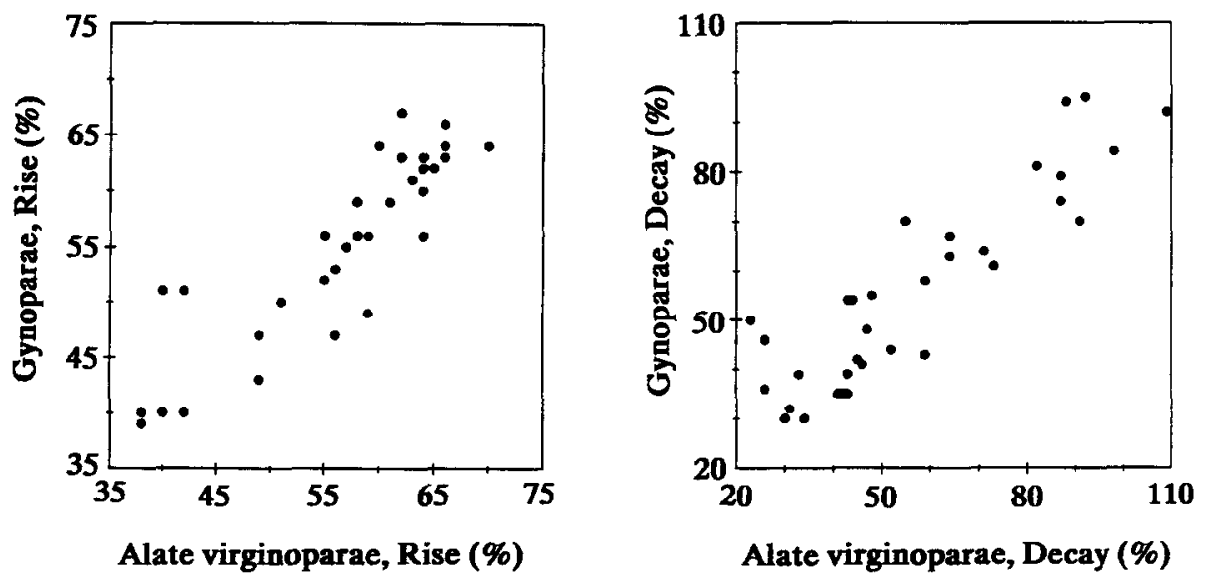

FIGURE 3. Comparison of (left) mean rise and (right) mean decay parameters of EAG responses from alate virginoparae and gynoparae to 33 test volatiles. 


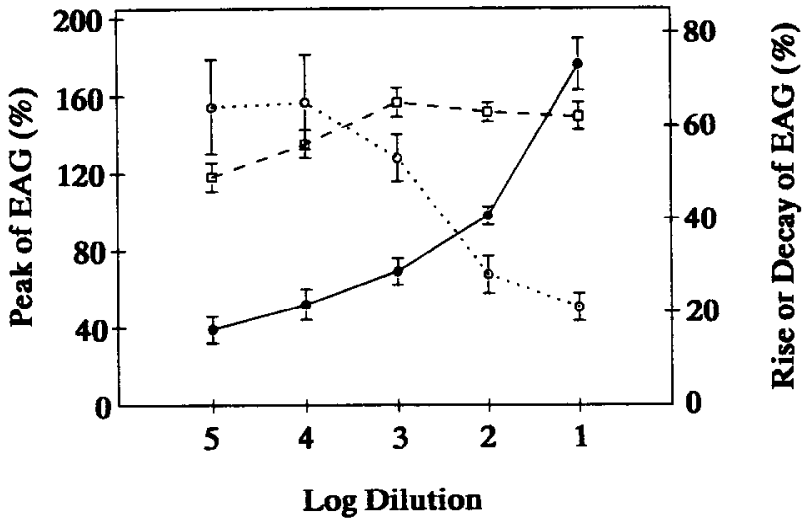

FIGURE 5. Dose-response relationship for normalised EAG peak responses (left Y-axis, mean $\pm 95 \%$ C.I., $n=9-15$ ) of alate virginoparae to $(E)$-2-hexenal (- - ). Dose-response relationships for rise $(--\square--)$ and for decay $(--\bigcirc \ldots)$ of the same EAGs (right $\mathrm{Y}$-axis, mean $\pm 95 \%$ C.I. $)$.

(Fig. 5). However, rise is similar for EAGs evoked by (E)-2-hexenal (2) at $\log 1-3$ dilutions while decay appears more sensitive and decreases rapidly at higher concentrations. The similarities between rise and decay parameters recorded from the different aphid forms is facilitated by the similarities in peak values.

Antennal responses from alate virginoparae and gynoparae to paraffin oil extracts of bean and spindle leaves are identical. There were no significant differences between the rise and decay parameters while Fig. 6 compares the normalised EAG peak responses.

\section{DISCUSSION}

The similarities between the absolute EAG peak size (in $\mu \mathrm{V}$ ), as well as the rise and decay parameters, of antennae from alate virginoparae and gynoparae to the standard $(E)$-2-hexenal indicate similar olfactory sensitivities to this volatile. These similarities also vindicate the use of this compound as a standard against which responses to other volatiles can be reliably compared. EAGs to the other thirty four volatiles support the contention that the peripheral olfactory perception

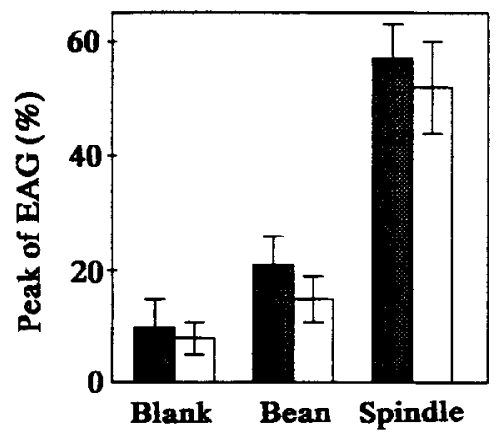

FIGURE 6. Comparison of the normalised EAG peak responses from alate virginoparae (hatched, $n=11$ ) and gynoparac (outline, $n=12$; mean $\pm 95 \%$ C.I.) evoked by paraffin oil extracts from broad bean ( $V . f a b a)$ and spindle (E. europaeus) leaves. of these two winged forms, which are morphologically the same but reproductively and behaviourally different (Hardie, 1980), is identical. Both forms are attracted to the odour of leaves from their respective hosts. Nottingham et al. (1991) reported attraction of alate virginoparae to bean leaf volatiles but could not find an attraction of gynoparae to spindle leaf volatiles in a linear track olfactometer. However, using the same clone of $A$. fabae in a Pettersson olfactometer, $\mathbf{R}$. Isaacs (pers. comm.) has found attraction to spindle leaves collected from the field during autumn when gynoparae are flying. The utilisation of different host plants does not appear to be assisted by olfaction at the peripheral level although central nervous system integration of olfactory information may provide distinction between odours emanating from the different hosts.

Results from the paraffin oil extracts of the summer host plant, broad bean, and the winter host, spindle, reveal only that a lesser EAG response is elicited to bean. This observation is undoubtedly due to a larger amount of volatiles extracted from the spindle and this was detectable by the human nose. The similarity between alate virginoparae and gynoparae further substantiate the conclusion that these clonal aphid forms can not distinguish between the odours of their relevant host plants at the peripheral sensory level.

Bromley and Anderson (1982) indicated that single cell recordings from primary rhinaria of greenhousereared currant-lettuce aphid, Nasonovia ribis-nigri, differed between the summer and autumn and postulated that this may be a change associated with the host-alternating life cycle (between lettuce, Lactuca spp and currant, Ribes spp). These observations are not supported by the present recordings from whole antennae although it is appreciated that the sensitivities of individual olfactory neurones (i.e. with different central projections) may differ between alate virginoparae and gynoparae and remain undetected in EAG responses. There is currently no way of electrophysiologically recording from positionally identified, individual antennal sensory neurones in aphids.

The finding that olfactory perception by apterous virginoparac is almost identical to the alate forms is somewhat surprising as the winged forms possess secondary rhinaria on the third, and sometimes on the fourth antennal segments, while the apterae do not (Jones, 1944; Hardie, 1980; Hardie et al., 1994). This result is in contrast to earlier reports of heightened EAG responses in winged virginoparae of Sitobion avenae compared with apterous forms (Yan and Visser, 1982). The secondary rhinaria do possess sensory neurones that respond to plant volatiles (Wadhams and Woodcock, quoted in Pickett et al., 1992) but their contribution to the overall EAG and to behaviour remains to be determined in female aphids although in males they provide the major olfactory organs for detection of sex pheromones (Pettersson, 1971; Marsh, 1975; Hardie et al., 1994). 

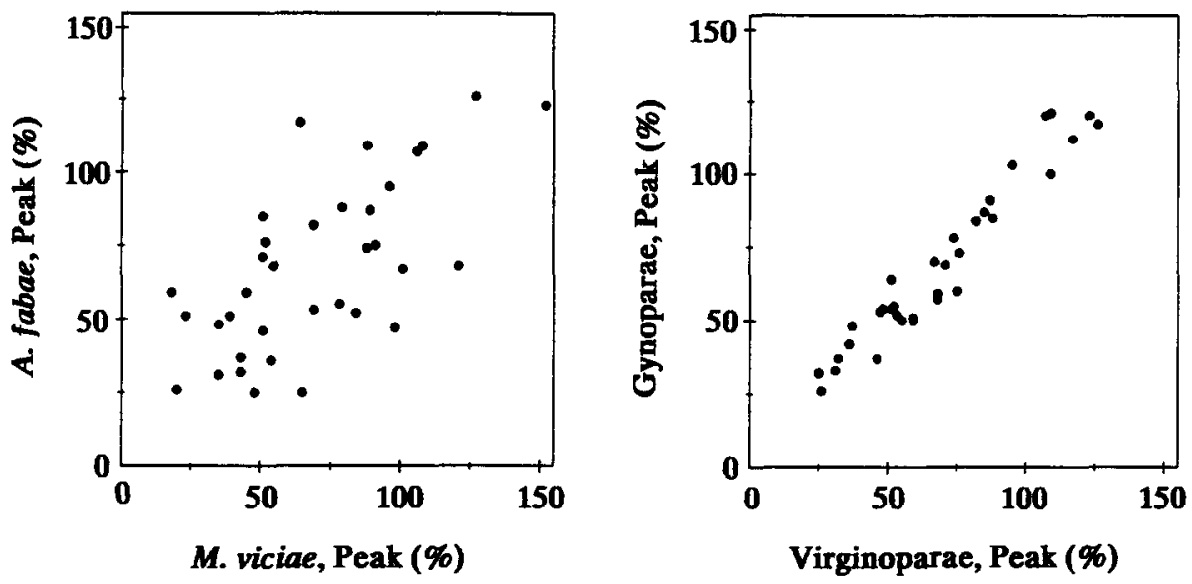

FIGURE 7. Comparison of mean normalised EAG peak responses to 35 test volatiles: (left) between $M$. viciae apterous virginoparae (Visser and Piron, 1994b) and $A$. fabae alate virginoparae and (right) between $A$. fabae gynoparae and alate virginoparae.

Recordings from single olfactory neurones associated with the proximal primary rhinarium of $A$. fabae alate virginoparae have shown that isothiocyanates differ in their potency such that 4-pentenyl isothiocyanate $>3$ butenyl isothiocyanate $>$ allyl isothiocyanate (Nottingham et al., 1991). The overall antennal response represented by the EAG broadly agrees with this finding with 4-pentenyl isothiocyanate $=3$-butenyl isothiocyanate $>$ allyl isothiocyanate although at the single cell level the latter compound appeared to have no effect.

It is also of interest to compare odour perception with a non-host-alternating aphid species, the vetch aphid Megoura viciae which utilises, throughout the year, a number of the leguminous host plants also associated with $A$. fabae. EAGs have been recorded from apterous M. viciae (Visser and Piron, 1994b) in exactly the same way as for $A$. fabae in the present study. From this $M$. viciae data set the same 35 stimuli reported in Fig. 2, at the same $\log 2$ dilution, were selected and used for comparison of mean normalised EAG peak responses between $M$. viciae and alate virginoparae of $A$. fabae (Fig. 7). In addition, Fig. 7 shows the comparison for gynoparae and alate virginoparae of $A$. fabae (the same data as Fig. 2). It is clear that the inter-species variation, $M$. viciae vs $A$. fabae, is much larger than the intra-specific variation, $A$. fabae gynoparae vs alate virginoparae.

In summary, it appears that although genetically identical summer (alate and apterous virginoparae) and autumn (gynoparae) forms of $A$. fabae have different host plant requirements as adults their peripheral olfactory perception is identical. This perception differs from a different aphid species, i.e. $M$. viciae, even though the two species may share the same host plant as larvac and adults. However, the inter-species comparison was drawn from aphids reared on different varieties of broad bean, namely the tick bean and cv. Minica, and it is known that EAGs differ between insects fed on different plants as shown for the Colorado potato beetle, Leptinotarsa decemlineata (De Jong et al., 1988). The effects of plant material, on which the aphids are reared, on the aphids' perception of volatiles requires investigation.

\section{REFERENCES}

Åhman I., Weibull J. and Pettersson J. (1985) The role of plant size and plant density for host finding in Rhopalosiphum padi (L.) (Hem.: Aphididae). Swedish J. agric. Res. 15, 19-24.

Boeckh J., Kaissling K.-E. and Schneider D. (1965) Insect olfactory receptors. Cold Spring Harb. Symp. Quant. Biol. 30, 263-280.

Bromley A. K. and Anderson M. (1982) An electrophysiological study of olfaction in the aphid, Nasonovia ribis-nigri. Ent. exp. appl. 32, 101-110.

Chapman R. F., Bernays E. A. and Simpson S. J. (1981) Attraction and repulsion of the aphid, Cavariella aegopodii, by plant odours. $J$. Chem. Ecol. 7, 881-888.

Dawson G. W., Griffiths D. C., Pickett J. A., Wadhams L. J. and Woodcock C. M. (1987) Plant-derived synergists of alarm pheromone from turnip aphid, Lypaphis (Hyadaphis) erisimi (Homoptera, Aphididae). J. Chem. Ecol. 13, 1663-1671.

De Jong R., Visser J. H., Van Strien E. A. and Nijhof C. M. (1988) Effects of feeding experience on host odour perception in the Colorado potato beetle. In R. de Jong, Host odour recognition by the Colorado potato beetle. $\mathrm{PhD}$ thesis, Wageningen Agricultural University, pp 51-60.

Dickens J. C., Visser J. H. and Van der Pers J. N. C. (1993) Detection and deactivation of pheromone and plant odor components by the beet armyworm, Spodoptera exigua (Hübner) (Lepidoptera: Noctuidae). J. Insect Physiol. 39, 503516.

Dixon A. F. G. (1981) The life-cycle and host preferences of the bird cherry-oat aphid, Rhopalosiphum padi L., and their bearing on theories of host alternation in aphids. Ann. appl. Biol. 68, 135-147.

Guldemond A. (1990) On aphids, their host plants and speciation, a biosystematic study of the genus Cryptomyzus (Homoptera, Aphididae). Ecol. Entomol. 5, 43-51.

Hardie J. (1980) Reproductive, morphological and behavioural affinities between the alate gynopara and virginopara of the aphid, Aphis fabae. Physiol. Entomol. 5, 385-396.

Hardie J. (1981) The effect of juvenile hormone on host-plant preference in the black bean aphid, Aphis fabae. Physiol. Entomol. 6, 369-374.

Hardie J. and Glascodine J. (1990) Polyphenism and host-plant preference in the black bean aphid, Aphis fabae Scop. Acta Phyto. Ent. Hung. 25, 323-330.

Hardie J., Visser J. H. and Piron P. G. M. (1994) Perception of volatiles associated with sex and food by different adult forms of the black bean aphid, Aphis fabae. Physiol. Entomol. 19, in press. 
Hille Ris Lambers D. (1966) Polymorphism in the Aphididae. Annu. Rev. Ent. 11, 47-78.

Jones M. G. (1944) The structure of the antenna of Aphis (Doralis) fabae Scopoli, and of Melanoxantherum salicis L. (Hemiptera), and some experiments on olfaction responses. Proc, R. Ent. Soc. Lond. (A) 19, 13-22.

Kennedy J. S. and Booth C. O. (1951) Host alternation in Aphis fabae Scop. I. Feeding preferences and fecundity in relation to age and kind of leaves. Ann. appl. Biol. 38, 25-64.

Kennedy J. S., Booth C. O. and Kershaw W. J. S. (1959) Host finding by aphids in the field. I. Gynoparae of Myzus persicae. Ann. appl. Biol. 47, 410-423.

Marsh D. (1975) Responses of male aphids to the female sex pheromone in Megoura viciae Buckton. J. Ent. (A) 50, 43-64.

Moran N. (1992) The evolution of aphid life cycles. Annu. Rev. Ent. 37, 321-348.

Nottingham S. F. and Hardie J. (1993) Flight behaviour of the black bean aphid, Aphis fabae, and the cabbage aphid, Brevicoryne brassicae, in host and non-host plant odour. Physiol. Entomol. 18, 389-394.

Nottingham S. F., Hardie J., Dawson G. W., Hick A. J., Pickett J. A., Wadhams L. J. and Woodcock C. M. (1991) Behavioural and electrophysiological responses of aphids to host and nonhost plant volatiles. J. Chem. Ecol. 17, 1231-1242.

Pettersson J. (1971) An aphid sex attractant II. Histological, ethological and comparative studies. Ent. Scand. 2, 81-93.

Pickett J. A., Wadhams L. J., Woodcock C. M. and Hardie J. (1992) The chemical ecology of aphids. Annu. Rev. Ent. 37, 67-90.

Van Giessen W. A., Peterson J. K. and Barnett O. W. (1992) Electroantennogram responses of aphids to plant volatiles and alarm pheromone. Proc. 8th Int. Symp. Insect-Plant Relationships. (Eds Menken S. J. B., Visser J. H. and Harrewijn P.), pp 117-118. Kluwer Academic Publ. Dordrecht.
Visser J. H. and Piron P. G. M. (1994a) Perception of plant odour components by the vetch aphid Megoura viciae: Shape characteristics of electroantennogram responses. Proc. exper. appl. Ent. N.E.V. Amsterdam, 5, 85-90.

Visser J. H. and Piron P. G. M. (1994b) Olfactory antennal responses to plant volatiles in apterous virginoparae of the vetch aphid Megoura viciae. Ent. exp. appl. In press.

Visser J. H. and Piron P. G. M. (1994c) Electroantennogram responses of the vetch aphid Megoura viciae to plant odor and pheromone components: shape characteristics show component-specific sensory transduction. J. Chem. Ecol. Submitted.

Visser J:H. and Taanman J. W. (1987) Odour-conditioned anemotaxis of apterous aphids (Cryptomyzus korschelti) in response to host plants. Physiol. Entomol. 12, 473-479.

Wadhams L. J. (1990) The use of coupled gas chromatography: electrophysiological techniques in the identification of insect pheromones. In Chromatography and Isolation of Insect Hormones and Pheromones (Eds McCaffery A. R. and Wilson I. D.) pp 289-298. Plenum, New York/London.

Wohlers P. and Tjallingii W. F. (1983) Electroantennogram responses of aphids to the alarm pheromone (E)- $\beta$-farnesene. Ent. exp. appl. 33, 79-82.

Yan F.-S. and Visser J. H. (1982) Electroantennogram responses of the cereal aphid Sitobion avenae to plant volatile components. Proc. 5th Int. Symp. Insect-Plant Relationships, Wageningen, 1982. (Eds Visser J. H. and Minks A. K.) pp 387-388. Pudoc, Wageningen.

Acknowledgements - Jim Hardie was supported by a fellowship from the Biotechnology and Biological Sciences Research Council. We thank A. J. Hick for synthesizing two of the isothiocyanates and A. M. van Oosten (TNO) for supplying the farnesenes. 